\title{
Transcription Factor-DNA Binding Motifs in Saccharomyces cerevisiae: Tools and Resources
}

\author{
Joshua L. Schipper and Raluca M. Gordân ${ }^{1}$ \\ Department of Biostatistics and Bioinformatics, Center for Genomic and Computational Biology, Duke University, \\ Durham, North Carolina 27708
}

\begin{abstract}
The DNA binding specificity of transcription factors (TFs) is typically represented in the form of a position weight matrix (PWM), also known as a DNA motif. A PWM is a matrix that specifies, for each position in the DNA binding site of a TF, the "weight" or contribution of each possible nucleotide. DNA motifs can be derived from various types of TF-DNA binding data, from small collections of known TF binding sites to large data sets generated using high-throughput technologies. One drawback of this simple model of DNA binding specificity is that it makes the implicit assumption that individual base pairs within a TF binding site contribute independently to the TF-DNA binding affinity. Although this assumption does not always hold, PWM models have been shown to provide reasonable approximations to the DNA binding specificity, and they are still widely used in practice. DNA motifs are currently available for more than 150 Saccharomyces cerevisiae TFs. Here, we briefly describe how these models are built, we provide information on databases containing DNA motifs for S. cerevisiae TFs, and we introduce guidelines on how to interpret the motifs and use them in practice to generate hypotheses about transcriptional regulatory regions.
\end{abstract}

\section{WHAT IS A TRANSCRIPTION FACTOR-DNA BINDING MOTIF?}

A DNA binding motif, or position weight matrix (PWM), is a simple and intuitive model for representing the DNA binding preferences of a transcription factor (TF). There are several accepted definitions of PWMs, depending on what the entries in the matrix represent (Stormo 2000, 2013). Typically, a PWM model $M$ is defined as a matrix with four rows (corresponding to the four nucleotides) and $W$ columns (where $W$ is the width of the TF binding site). Each element $M(i, j)$ in the matrix represents the probability of seeing nucleotide $i$ at position $j$ in the binding site (Fig. 1A). This type of matrix is also known as a position frequency matrix (PFM) or position-specific scoring matrix (PSSM). In other definitions of the PWM, entries in the matrix may correspond to the energy contribution of each nucleotide at each position, the binding affinity for each nucleotide at each position, or the log ratio of the probability of each nucleotide at each position in the TF binding site relative to its background probability. The exact meaning of the entries in a PW will affect how the PWM is used to score putative TF binding sites, as described below.

\section{SCORING A PUTATIVE TF BINDING SITE ACCORDING TO A DNA MOTIF}

Given a putative TF binding site $S$ and a DNA motif $M$ containing the probabilities of each nucleotide at each position, we can compute a "score" for site $S$ as

\footnotetext{
${ }^{1}$ Correspondence: raluca.gordan@duke.edu

(C) 2016 Cold Spring Harbor Laboratory Press

Cite this introduction as Cold Spring Harb Protoc; doi:10.1101/pdb.top080622
} 
A

DNA motif $M$

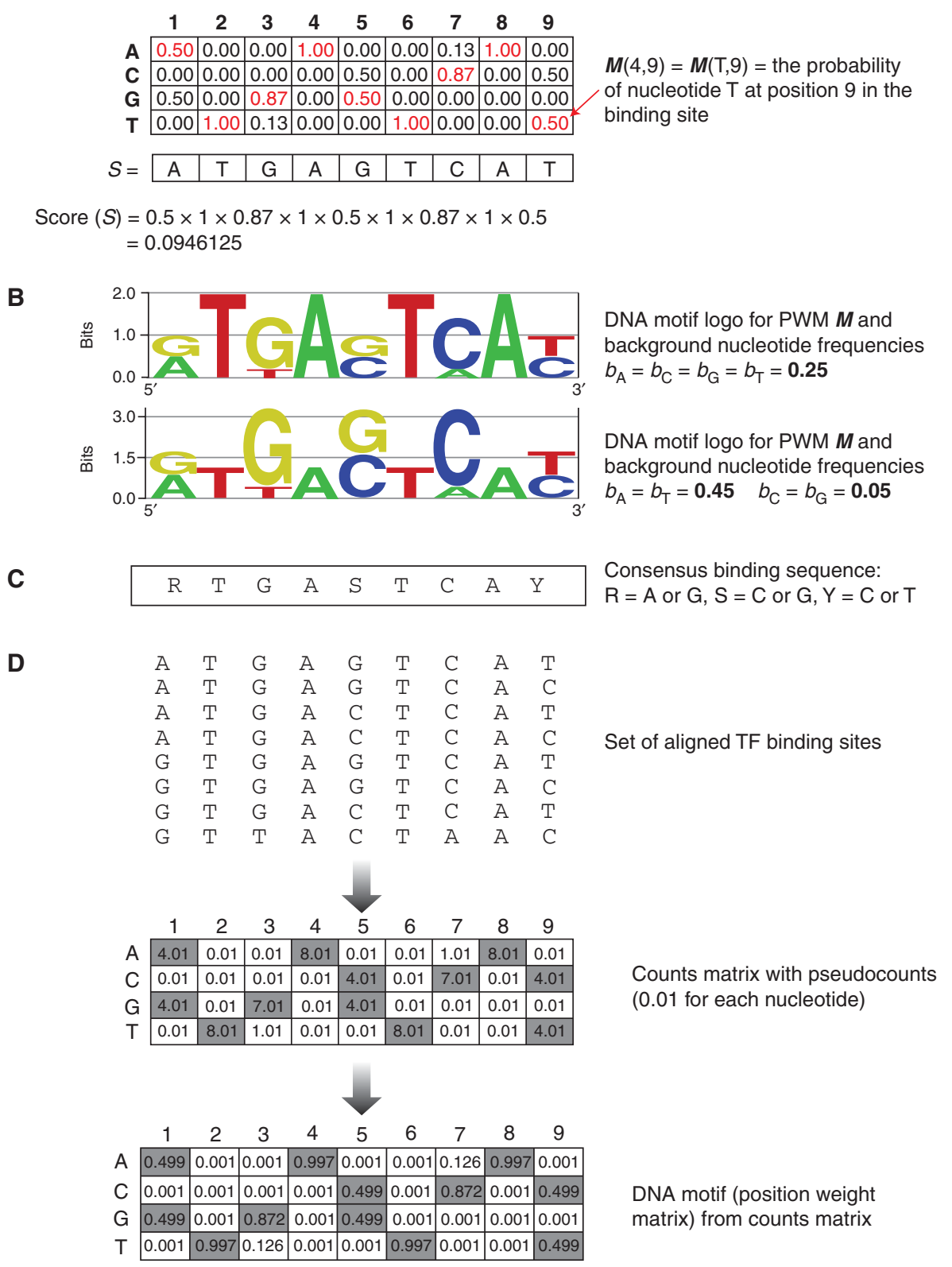

FIGURE 1. TF-DNA binding models. (A) Representation of a TF-DNA binding motif as a position weight matrix (PWM). (B) DNA motif logos generated using the enoLOGOS web server (Workman et al. 2005) for the PWM in $A$ using a uniform background distribution $b_{\mathrm{A}}=b_{\mathrm{C}}=b_{\mathrm{G}}=b_{\mathrm{T}}=0.25$ (top logo) or $b_{\mathrm{A}}=b_{\mathrm{T}}=0.45, b_{\mathrm{C}}=b_{\mathrm{G}}=0.05$ (bottom logo). (C) Consensus binding sequence derived from DNA motif in $A$. (D) Building a DNA motif from a set of aligned TF binding sites.

$$
\operatorname{Score}(S)=P(S \mid M)=\prod_{i=1}^{W} M\left(S_{i}, i\right)
$$

where $M\left(S_{i}, i\right)$ is the probability of seeing nucleotide $S_{i}$ at position $i$ in the binding site (Fig. 1A). If we consider the PWM as a generative model (i.e., a model that generates binding sites for the TF of interest), then we can interpret Score $(S)$ as the probability of generating binding site $S$ from the PWM model $M$ (i.e., $P(S \mid M)$ ). Because the PWM model assumes that the contributions of nucleotides at different positions in the binding site are independent of each other, the probability of generating site 
$S$ from the PWM is simply the product of generating, independently, each nucleotide $S_{i}$ at each position $i$ (Fig. 1A). In the case of PWMs where the entries represent energies, the product in Equation 1 should be replaced by a sum, as each nucleotide in a binding site contributes additively to the binding energy for that site.

An alternative way of scoring putative TF binding sites using a PWM is to use the log-likelihood ratio (LLR). Let $B$ be a background model representing the nucleotide frequencies in "background" or random DNA sequences (i.e., sequences that are not binding sites for the TF of interest). The LLR score of a putative DNA site $S$ is the natural logarithm of the ratio between the probability of generating $S$ from the PWM model $M$, and the probability of generating $S$ from the background model $B$ :

$$
\operatorname{Score}_{\mathrm{LLR}}(S)=\ln \frac{P(S \mid M)}{P(S \mid B)}=\ln \prod_{i=1}^{W} \frac{M\left(S_{i}, i\right)}{b_{S_{i}}}=\sum_{i=1}^{W} \ln \frac{M\left(S_{i}, i\right)}{b_{S_{i}}}
$$

where $b_{j}$ is the frequency of nucleotide $j$ in background DNA sequences. A positive LLR score indicates that site $S$ is more likely to be a TF binding site than to be part of the background DNA. A negative LLR score indicates that site $S$ is more likely to come from background DNA than to be a binding site for a TF with the DNA motif $M$.

For both the PWM score (Equation 1) and the LLR score (Equation 2), higher scores correspond to sites that are more likely to be bound by the TF. In the case of PWMs where the entries in the matrix represent log ratios of generating each nucleotide from the motif model versus a background model, these entries are equal to $\ln \left(M(i, j) / b_{i}\right)$, and the score according to such a PWM is exactly the log-likelihood ratio score described in Equation 2. This type of matrix is sometimes referred to as a "log-odds" matrix.

\section{VISUALIZING TF-DNA BINDING MOTIFS}

TF-DNA binding motifs can be visualized using motif logos (Fig. 1B). A motif logo contains as many positions as the DNA motif. At each position, the relative heights of the letters are proportional to their probabilities, as defined by the PWM. The total height of each column $j$ is the information content (IC) of that column, represented as the number of bits of information and defined as

$$
\mathrm{IC}_{j}=\sum_{i \in\{A, C, G, T\}} M(i, j) \times \log _{2} \frac{M(i, j)}{b_{i}},
$$

where $M(i, j)$ is the probability of nucleotide $i$ at position $j$ in the PWM, and $b_{i}$ is the background frequency of nucleotide $i$. Intuitively, high information content positions are very stringent- that is, mutations in TF binding sites that occur at positions with high IC will significantly affect the binding affinity. Low information content corresponds to a more degenerate position, where several nucleotides are accepted in the TF binding site; mutations at positions with low IC have a lesser effect on the binding affinity.

Publicly available software and web tools for generating motif logos include the following:

- enoLOGOS: http://www.benoslab.pitt.edu/cgi-bin/enologos/enologos.cgi (Workman et al. 2005) and

- WebLogos: http://weblogo.berkeley.edu/logo.cgi (Crooks et al. 2004)

Such software tools allow users to build logos for both DNA and protein sequences. Each software tool has several user-defined parameters and can accept DNA motifs in different formats, including position frequency matrices (e.g., the PWM in Fig. 1A), aligned TF binding sites, or PWMs downloaded directly from DNA motif databases (such as TRANSFAC; Matys et al. 2006). Refer to the manual of each tool for more details. 
An important parameter that the user should always set when generating DNA motif logos is the background nucleotide distribution. Different background distributions will lead to different motif logos, as shown in Figure 1B. For generating DNA motif logos, the most widely used background nucleotide distribution is the uniform distribution: $b_{\mathrm{A}}=b_{\mathrm{C}}=b_{\mathrm{G}}=b_{\mathrm{T}}=0.25$. In some DNA motif discovery tools, such as enoLOGOS (Workman et al. 2005), this distribution can be specified by setting the GC content parameter (called "\%GC" in enoLOGOS) to 50\%. This type of background model will lead to DNA motifs where the information content at each position (i.e., the $y$-axis in the motif logo) is between 0 (the most degenerate position possible) and 2 (the most stringent position possible). Figure 1B shows examples of motif logos generated using the same PWM (the one in Fig. 1A) but different background nucleotide distributions (a uniform distribution in the top logo, and a highly skewed distribution, with GC content of 10\%, in the bottom logo). According to the DNA motif generated using the uniform background distribution, positions 2, 4, 6, and 8 are very stringent (i.e., not degenerate), and $\mathrm{A}$ or $\mathrm{T}$ nucleotides at these positions seem to be highly preferred. When a background GC content of $10 \%$ is used, positions 2, 4, 6, and 8 appear more degenerate, and their height relative to other positions decreases. Intuitively, this is due to the fact that when the background GC content is $10 \%$, the general frequencies of $\mathrm{A}$ and $\mathrm{T}$ nucleotides are very high (the AT content is $90 \%$ ), so even DNA sites that are not putative TF binding sites will contain a large number of A and T nucleotides simply by chance. Thus, having an A or T in a putative TF binding site is not as significant as in the case of a uniform background distribution. In general, we recommend using a uniform background nucleotide distribution when generating DNA motif logos.

\section{FROM DNA MOTIFS TO CONSENSUS SEQUENCES}

DNA motifs can be used to derive consensus binding sequences, which use the four DNA bases and a set of IUPAC (International Union and Pure and Applied Chemistry) codes to specify the most common nucleotides at each position in a TF binding site (Fig. 1C). Consensus sequences have an intuitive interpretation: The TF has a high affinity for sequences matching the consensus and a low affinity for sequences that deviate from the consensus. However, this separation into binding sites and nonbinding sites is artificial because TFs bind a wide range of DNA sites with a continuum of binding affinities (Siggers and Gordân 2014).

The PWM model is a generalization of the consensus sequence model. The advantage of the PWM is that it provides position-specific penalties for deviations from the consensus; thus, when comparing a putative TF binding site to a PWM (as opposed to a consensus sequence) not all mismatches are treated equally, and some mismatches are more detrimental than others. For example, the DNA site ATGAGTCAT has the highest possible score according to the PWM in Figure 1A, and it matches the consensus sequence shown in Figure 1C (RTGASTCAY). If we mutate the G at position 3 to either an $\mathrm{A}$ or a $\mathrm{T}$, then we obtain sites that do not match the consensus and thus would not be considered putative TF binding sites according to the consensus model. However, if we use the PWM model, then the score for ATGAGTCAT will be higher than the score of both mutated sites (ATAAGTCAT and ATTAGTCAT), but the two mutated sites are considered of different affinity, with ATTAGTCAT being preferred over ATAAGTCAT because it has a higher PWM score: Score LLR $_{\text {LT }}($ ATGGTCAT) $=$ 10.12, Score $_{\text {LLR }}($ ATTAGTCAT $)=8.05$, and $\operatorname{Score}_{\text {LLR }}($ ATAAGTCAT $)=5.65$.

\section{BUILDING DNA BINDING MOTIFS}

Generating TF-DNA binding motifs depends, in part, on the DNA binding data available for the TF of interest. The simplest case is when we are given a set of aligned, known TF binding sites; such data are available from databases such as TRANSFAC (Matys et al. 2006) and JASPAR (Mathelier et al. 2014). Given this set of aligned binding sites, PWM models can be derived by computing the counts for each nucleotide at each position and then transforming the counts into frequencies by normalizing 
the values in each column to sum to 1 (i.e., by dividing each item in the matrix by the number of sites used to build the PWM) (Fig. 1D). Typically, before normalizing each column, a small number (e.g., 0.01 ) representing pseudocounts is added to each entry in the matrix to avoid occurrences of zero values in the PWM. Such zero probabilities are unlikely because we cannot be $100 \%$ certain that a particular nucleotide will never occur in a binding site for a particular TF. In addition, zero values may confound the results of applications that use PWMs. For example, when computing log-likelihood ratio scores using PWMs with zero entries, we run into the problem of having to compute $\log (0)$, which is not defined. Setting the exact pseudocounts to add when deriving a PWM is somewhat ad hoc. In general, small values such as 0.001 or 0.01 are used; however, when the number of aligned binding sites is very large (hundreds or thousands of sites), larger pseudocounts are recommended to avoid having very small values in the PWM. A value close to $0.1 \%$ of the total number of sites often yields reasonable results.

When aligned TF binding sites are not available, one can derive PWMs from sets of genomic or artificial sequences bound by the TF of interest (e.g., sequence reported to be bound according to ChIP-chip [Ren et al. 2000] or ChIP-seq [Johnson et al. 2007] data, or promoter sequences of genes believed to be regulated by a common TF). Once a set of "bound" DNA sequences has been identified, one can use motif discovery tools to identify PWMs that are enriched in those sequences. Hundreds of motif discovery methods have been developed to date, but few of them are easily accessible to researchers without a strong computational background. One of the easily accessible and wellsupported motif discovery tools is MEME (Bailey and Elkan 1994). MEME is widely used and can be applied to relatively small sets (tens or hundreds) of DNA sequences (http://meme.nbcr.net/meme/), as well as high-throughput data generated using ChIP-seq assays (http://meme.nbcr.net/meme/ cgi-bin/meme-chip.cgi). The MEME Suite also provides additional tools for analysis of TF-DNA binding data.

Figure 2 shows an example of running MEME to find overrepresented DNA motifs in ChIP-chip data for the Saccharomyces cerevisiae TF Gcn4 (Harbison et al. 2004). A total of 58 sequences with ChIP-chip $p$-value $<0.001$ were used in this analysis. When run with the default settings, MEME found three DNA motifs (Fig. 2). For each motif, the tool also reported the number of DNA sites containing matches to that motif, as well as the statistical significance of the motif, represented as an

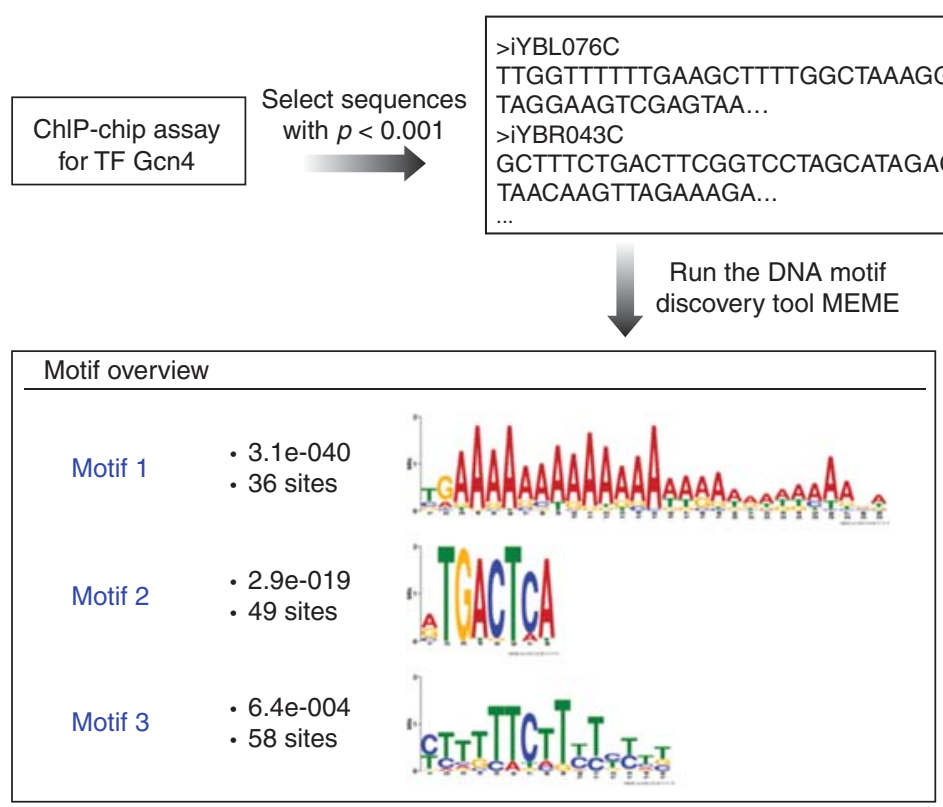

FIGURE 2. Example of running the MEME motif discovery tool on a set of DNA sequences bound by Saccharomyces cerevisiae TF Gcn4, according to ChIP-chip (chromatin immunoprecipitation with DNA microarray) data (Harbison et al. 2004). 
$E$-value. Intuitively, the $E$-value can be interpreted as the number of motifs as good as or better than the motif of interest, identified in a set of random DNA sequences (for more details, see the documentation provided with the MEME tool). Interpreting the results of a motif discovery tool is not trivial. In general, motifs with lower $E$-values (i.e., with higher statistical significance) are preferred. However, as can be seen in Figure 2, motifs with very low E-values, such as Motif 1, sometimes capture DNA sequences that are common in the set of input DNA sequences but are unlikely to represent TF binding sites. In this example, Motif 2 is the correct motif for TF Gcn4; it has a high significance $(E$-value $=2.9 \mathrm{e}-019)$ and a large number of DNA sites ( 49 sites in the 58 sequences given as input to the algorithm). Motif 3 also has a large number of occurrences, but its significance is relatively low $(E$-value $=6.4 \mathrm{e}-4)$, which makes it unlikely to be the DNA motif for the TF tested in the ChIP-chip experiment (i.e., TF Gcn4). Further information about the DNA motifs identified by MEME is available in the results provided by the algorithm.

\section{REPOSITORIES OF DNA BINDING MOTIFS FOR S. Cerevisiae TFs}

DNA motifs for more than 150 S. cerevisiae TFs are currently available in online databases and in various publications.

- Saccharomyces Genome Database (SGD): http://www.yeastgenome.org (Cherry et al. 2012). For each TF, SGD contains the DNA binding motif reported in the YeTFaSCo database (http:// yetfasco.ccbr.utoronto.ca) (de Boer and Hughes 2012), as well as links to other DNA motif databases. Importantly, the YeTFaSCo database also provides expert curated information for the available DNA motifs.

- DNA motifs for S. cerevisiae TFs have been derived from large collections of ChIP-chip experiments by Harbison et al. (2004) and MacIsaac et al. (2006).

- TRANSFAC: www.biobase-international.com/product/transcription-factor-binding-sites (Matys et al. 2006). For each TF, the TRANSFAC database contains consensus binding sequences, motif logos, and PWM descriptions (typically as counts instead of frequencies), as well as the exact binding sites used to generate the PWMs, when available. The PWMs reported in TRANSFAC are sometimes derived from small-scale assays (such as electrophoretic mobility shift assays or footprinting) or from small collections of binding sites reported in the literature. Such PWMs may be biased toward the high-affinity sites characterized previously and may not accurately reflect moderate affinity TF binding sites. We recommend using PWMs derived from higher-throughput experimental data such as in vivo ChIP-chip/ChIP-seq assays (Ren et al. 2000; Johnson et al. 2007) or in vitro protein-binding microarray (PBM) (Berger et al. 2006) or mechanically induced trapping of molecular interactions (MITOMI) (Maerkl and Quake 2007) experiments. Access to the latest version of the TRANSFAC database requires an annual subscription. Older versions are freely available.

- JASPAR: http://jaspar.genereg.net (Mathelier et al. 2014). For each TF, the JASPAR database contains motif logos and PWM descriptions (represented as counts), as well as the exact binding sites used to generate the PWMs, when available. The type of data used to generate each PWM is specified. Compared to TRANSFAC, the JASPAR database contains information for fewer TFs; however, the data available in JASPAR is typically curated and restricted to highquality motifs. In addition, a unique feature of JASPAR PWMs (compared with other sources of $S$. cerevisiae motifs described here) are the links to the TFBSshape database (Yang et al. 2014), which contains information on the DNA structure of binding sites for certain TFs.

- UniPROBE: http://the_brain.bwh.harvard.edu/uniprobe/ (Robasky and Bulyk 2011). The UniPROBE database contains TF-DNA binding data and PWMs derived from in vitro universal PBM experiments (Berger et al. 2006). Compared with other assays used to generate DNA motif models, universal PBM assays are unbiased and comprehensive, as they measure the binding 
specificity of a TF for all possible ungapped 10-mers and a large number of 10-mers containing gaps (for details on the experimental design, see Berger et al. 2006; Berger and Bulyk 2009). In addition, PBM data are advantageous in that they reflect the direct DNA binding specificity of the tested TF, in contrast to in vivo assays such as ChIP, for which both direct and indirect TF-DNA binding events are represented in the data. Gordân et al. (2009) performed a detailed analysis of direct and indirect TF-DNA binding for S. cerevisiae TFs by combining in vivo ChIP-chip data (Harbison et al. 2004) and PWMs derived from universal PBMs (Badis et al. 2008; Zhu et al. 2009).

- The PWMs available in UniPROBE were derived from the universal PBM data reported in a largescale study of yeast TFs (Zhu et al. 2009). Later, Gordân et al. (2011) added PBM data for 27 additional yeast TFs and reanalyzed the data reported by Zhu et al. (2009), as well as the PBM data of Badis et al. (2008) and the MITOMI data of Fordyce et al. (2010). In total, in vitro TF-DNA binding data for 150 yeast TFs was curated in Gordân et al. (2011). The quality of each motif was assessed based on the available in vitro and in vivo data and, for each TF, the DNA motif that best explains the in vivo binding data for that factor was reported. These curated DNA motifs are available as Data File S1 in Gordân et al. (2011). In addition, this curated collection reports, for each of the 150 TFs analyzed in the study, the binding specificities for all possible 8-mers, derived from the universal PBM data. For each TF and each 8-mer, an enrichment score (or E-score) is reported, which reflects the enrichment of the 8-mer among the DNA probes bound with high affinity in the PBM assay for that TF. The E-score is a modified form of the Wilcoxon-MannWhitney statistic and ranges from -0.5 (least favored sequence) to +0.5 (most favored sequence), with values $>0.35$ corresponding, in general, to sequence-specific DNA binding of the tested TF (Berger et al. 2006).

Importantly, it has recently been shown that about one-third of S. cerevisiae TFs have two distinct modes of binding DNA, described by two PWMs (typically called "primary" and "secondary" motifs; Gordân et al. 2011). Such motifs were derived from PBM data for yeast TFs and reported in Data File S1 in Gordân et al. (2011). TFs with multiple modes of binding have long been reported in the literature, but the large number of such TFs has recently been revealed by large-scale in vitro studies. The distinct modes of DNA binding are believed to reflect slightly different conformations of the TFs bound to DNA (see Gordân et al. 2011 for more details and information on yeast TFs with multiple modes of DNA binding). When using DNA motifs to identify putative binding sites for such TFs, it is important to take into account the primary and the secondary motifs, as both motifs have been shown to be relevant for TF-DNA binding in the cell (Gordân et al. 2011).

DNA motifs can be used to scan genomic regions of interest (such as gene promoters) to identify putative TF binding sites. Given a TF-DNA binding motif $M$ of width $W$ and a DNA sequence $X$ of length $L$, we can use the DNA motif to scan the sequence $X$ and score each site of size $W$ in the sequence using, for example, the LLR score. Figure 3A shows an example of scanning part of an intergenic yeast sequence (iYJL089W) using the LLR score with a DNA motif for TF Gcn4 and a uniform background distribution. In general, when scanning a sequence with a DNA motif to identify putative TF binding sites, we will report all sites for which the score represents at least a fraction $F$ of the score for the best possible site according to that DNA motif. The score of the best site can be computed by taking, from each PWM column, the entry containing the highest probability.

Most DNA motif databases mentioned above also provide tools for identifying putative TF binding sites using the reported motifs. For example, the JASPAR web server (Mathelier et al. 2014) contains a simple online tool that can be used to scan a FASTA-formatted DNA sequence using the PWM model (s) selected by the user. Only one parameter is required: the relative score threshold, which represents the ratio between the LLR score of a putative TF binding site versus the LLR score of the best site according to the selected PWM model (i.e., the $F$ fraction mentioned above and in Fig. 3A). Figure 3B 
A

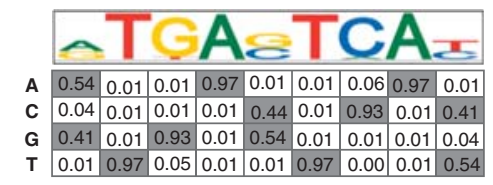

>IYJL089W

TGAACTCGACCTTCTAACATTACGCT CCTTCGTATTACTCATTCAGCTCTTC CTCTGATAGCAGTGAATTTTCGAGGG TCACGTCGTGACTCATATGCTTTCT

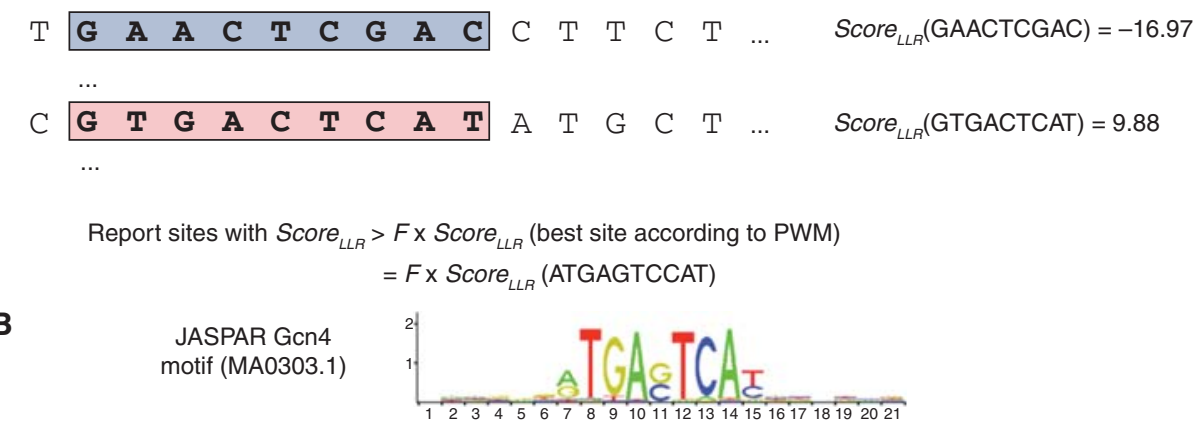

Sites identified by JASPAR with Score $_{L R}>0.8 \times$ Score $_{L R}$ (best site according to PWM):

\begin{tabular}{|l|l|l|l|l|l|l|l|}
\hline Model ID & Model name & Score & Relative score & Start & End & Strand & Predicted site sequence \\
\hline MA0303.1 & GCN4 & 10.806 & 0.860456995001173 & 28 & 48 & -1 & AGCTGAATGAGTAATACGAAG \\
\hline MA0303.1 & GCN4 & 10.312 & 0.852906200383129 & 28 & 48 & 1 & CTTCGTATTACTCATTCAGCT \\
\hline MA0303.1 & GCN4 & 10.926 & 0.862291196122965 & 80 & 100 & -1 & AAGCATATGAGTCACGACGTG \\
\hline MA0303.1 & GCN4 & 14.012 & 0.909460734971718 & 80 & 100 & 1 & CACGTCGTGACTCATATGCTT \\
\hline
\end{tabular}

FIGURE 3. Identifying putative TF binding sites using a DNA motif. (A) A DNA motif for TF Gcn4 is used to identify putative Gcn4 binding sites, defined as sites with LLR score $>F \times$ the LLR score of the best DNA site according the motif (here, ATGAGTCAT). (B) Putative Gcn4 binding sites reported by JASPAR (Mathelier et al. 2014) using the Gcn4 motif MA0303.1 and a relative score cutoff $F=0.8$

shows the putative Gcn4 binding sites identified using the JASPAR Gcn4 motif and a relative score threshold of 0.8 .

Additional online tools for calling putative TFBSs using DNA motifs include the following:

- FIMO: http://meme.nbcr.net/meme/cgi-bin/fimo.cgi (part of the MEME Suite)

- PATSER: http://rsat.ulb.ac.be/patser_form.cgi (part of the RSA-tools suite)

Further information about these tools, their output, and the interpretation of the output is available on the tools' websites.

The 8-mer PBM data reported in Badis et al. (2008) and Zhu et al. (2009) and curated in Gordân et al. (2011) can also be used to identify putative TF binding sites. Briefly, one can scan the sequences of interest to search for 8 -mers with enrichment scores $(E$-scores $)>0.35$, which likely represent sequence-specific TF-DNA binding sites. For increased confidence, one can search for two consecutive, overlapping 8-mers with E-scores above a certain cutoff (as done, e.g., in Gordân et al. 2013).

\section{COMPUTING THE ENRICHMENT OF A DNA MOTIF IN A SET OF DNA SEQUENCES}

Certain motif discovery tools, such as MEME, automatically provide a measure of statistical significance for the identified DNA motifs. However, there are situations when a PWM for the TF of interest is already available, and we are interested in determining whether or not the PWM is enriched in a set of DNA sequences (e.g., in promoters of a set of co-regulated genes). The hypergeometric distribution (i.e., Fisher's exact test) can be used for this purpose. To apply this test, we first need to define a "foreground" set containing DNA sequences that are likely to be bound by the TF of interest, and a "background" set containing unbound sequences (e.g., if the foreground set contains promoters of 
co-regulated genes, the background set can be composed of randomly chosen promoters of other genes in the S. cerevisiae genome). Next, we use the DNA motif to scan each foreground and background sequence and determine how many of the sequences contain putative TF binding sites (at a specific relative score threshold $F$ ). Let $N$ be the total number of sequences, in both the foreground and background sets, $m$ the number of sequences containing putative TF binding sites, $n$ the number of foreground sequences, and $k$ the number of foreground sequences that contain putative TF binding sites. Then we can use the hypergeometric distribution (Equation 4) and compute the $p$-value as the cumulative probability $P(X \geq k)$ (a small $p$-value will indicate that the DNA motif is enriched in the foreground sequences compared to the background sequences):

$$
P(X=k)=\frac{\left(\begin{array}{c}
m \\
k
\end{array}\right)\left(\begin{array}{c}
N-m \\
n-k
\end{array}\right)}{\left(\begin{array}{c}
N \\
n
\end{array}\right)} \text {. }
$$

\section{REFERENCES}

Badis G, Chan ET, van Bakel H, Pena-Castillo L, Tillo D, Tsui K, Carlson CD, Gossett AJ, Hasinoff MJ, Warren CL, et al. 2008. A library of yeast transcription factor motifs reveals a widespread function for Rsc3 in targeting nucleosome exclusion at promoters. Mol Cell 32: 878-887. doi: 10.1016/j.molcel.2008.11.020.

Bailey TL, Elkan C. 1994. Fitting a mixture model by expectation maximization to discover motifs in biopolymers. Proc Int Conf Intell Syst Mol Biol 2: 28-36.

Berger MF, Bulyk ML. 2009. Universal protein-binding microarrays for the comprehensive characterization of the DNA-binding specificities of transcription factors. Nat Protoc 4: 393-411. doi: 10.1038/ nprot.2008.195.

Berger MF, Philippakis AA, Qureshi AM, He FS, Estep PW 3rd, Bulyk ML. 2006. Compact, universal DNA microarrays to comprehensively determine transcription-factor binding site specificities. Nat Biotechnol 24: 1429-1435. doi: 10.1038/nbt1246.

Cherry JM, Hong EL, Amundsen C, Balakrishnan R, Binkley G, Chan ET, Christie KR, Costanzo MC, Dwight SS, Engel SR, et al. 2012. Saccharomyces genome database: The genomics resource of budding yeast. Nucleic Acids Res 40: D700-D705. doi: 10.1093/nar/gkr1029.

Crooks GE, Hon G, Chandonia JM, Brenner SE. 2004. WebLogo: A sequence logo generator. Genome Res 14: 1188-1190. doi: 10.1101/gr.849004.

de Boer CG, Hughes TR. 2012. YeTFaSCo: A database of evaluated yeast transcription factor sequence specificities. Nucleic Acids Res 40: D169D179. doi: 10.1093/nar/gkr993.

Fordyce PM, Gerber D, Tran D, Zheng J, Li H, DeRisi JL, Quake SR. 2010. De novo identification and biophysical characterization of transcription-factor binding sites with microfluidic affinity analysis. Nat Biotechnol 28: 970-975. doi: 10.1038/nbt.1675.

Gordân R, Hartemink AJ, Bulyk ML. 2009. Distinguishing direct versus indirect transcription factor-DNA interactions. Genome Res 19: 20902100. doi: 10.1101/gr.094144.109.

Gordân R, Murphy KF, McCord RP, Zhu C, Vedenko A, Bulyk ML. 2011. Curated collection of yeast transcription factor DNA binding specificity data reveals novel structural and gene regulatory insights. Genome Biol 12: R125. doi: 10.1186/gb-2011-12-12-r125.

Gordân R, Shen N, Dror I, Zhou T, Horton J, Rohs R, Bulyk ML. 2013. Genomic regions flanking E-Box binding sites influence DNA binding specificity of bHLH transcription factors through DNA shape. Cell Rep 3: 1093-1104. doi: 10.1016/j.celrep.2013.03.014.

Harbison CT, Gordon DB, Lee TI, Rinaldi NJ, MacIsaac KD, Danford TW, Hannett NM, Tagne JB, Reynolds DB, Yoo J, et al. 2004. Transcriptional regulatory code of a eukaryotic genome. Nature 431: 99-104. doi: 10.1038/nature02800.
Johnson DS, Mortazavi A, Myers RM, Wold B. 2007. Genome-wide mapping of in vivo protein-DNA interactions. Science 316: 1497- 1502. doi: 10.1126/science.1141319.

MacIsaac KD, Wang T, Gordon DB, Gifford DK, Stormo GD, Fraenkel E. 2006. An improved map of conserved regulatory sites for Saccharomyces cerevisiae. BMC bioinformatics 7: 113, doi: 10.1186/1471-2105-7-113.

Maerkl SJ, Quake SR. 2007. A systems approach to measuring the binding energy landscapes of transcription factors. Science 315: 233-237. doi: 10.1126/science.1131007.

Mathelier A, Zhao X, Zhang AW, Parcy F, Worsley-Hunt R, Arenillas DJ, Buchman S, Chen CY, Chou A, Ienasescu H, et al. 2014. JASPAR 2014: An extensively expanded and updated open-access database of transcription factor binding profiles. Nucleic Acids Res 42: D142-D147. doi: $10.1093 / \mathrm{nar} / \mathrm{gkt} 997$.

Matys V, Kel-Margoulis OV, Fricke E, Liebich I, Land S, Barre-Dirrie A, Reuter I, Chekmenev D, Krull M, Hornischer K, et al. 2006. TRANSFAC and its module TRANSCompel: Transcriptional gene regulation in eukaryotes. Nucleic Acids Res 34: D108-D110, doi: 10.1093/nar/gkj143.

Ren B, Robert F, Wyrick JJ, Aparicio O, Jennings EG, Simon I, Zeitlinger J, Schreiber J, Hannett N, Kanin E, et al. 2000. Genome-wide location and function of DNA binding proteins. Science 290: 2306-2309. doi: 10.1126/science.290.5500.2306.

Robasky K, Bulyk ML. 2011. UniPROBE, update 2011: Expanded content and search tools in the online database of protein-binding microarray data on protein-DNA interactions. Nucleic Acids Res 39: D124-D128. doi: 10.1093/nar/gkq992.

Siggers T, Gordân R. 2014. Protein-DNA binding: Complexities and multiprotein codes. Nucleic Acids Res 42: 2099-2111. doi: 10.1093/nar/ gkt1112.

Stormo GD. 2000. DNA binding sites: Representation and discovery. Bioinformatics 16: 16-23.

Stormo GD. 2013. Modeling the specificity of protein-DNA interactions. Quant Biol 1: 115-130.

Workman CT, Corcoran DL, Ideker T, Stormo GD, Benos PV. 2005. enoLOGOS: A versatile web tool for energy normalized sequence logos. Nucleic Acids Res 33: W389-W392, doi: 10.1093/nar/gki439.

Yang L, Zhou T, Dror I, Mathelier A, Wasserman WW, Gordân R, Rohs R. 2014. TFBSshape: A motif database for DNA shape features of transcription factor binding sites. Nucleic Acids Res 42: D148-D155. doi: 10.1093/nar/gkt1087.

Zhu C, Byers KJ, McCord RP, Shi Z, Berger MF, Newburger DE, Saulrieta K, Smith Z, Shah MV, Radhakrishnan M, et al. 2009. High-resolution DNA-binding specificity analysis of yeast transcription factors. Genome Res 19: 556-566. doi: 10.1101/gr.090233.108. 


\section{Transcription Factor-DNA Binding Motifs in Saccharomyces cerevisiae: Tools and Resources}

Joshua L. Schipper and Raluca M. Gordân

Cold Spring Harb Protoc; doi: 10.1101/pdb.top080622

\begin{tabular}{cc}
$\begin{array}{c}\text { Email Alerting } \\
\text { Service }\end{array}$ & Receive free email alerts when new articles cite this article - click here. \\
\hline $\begin{array}{c}\text { Subject } \\
\text { Categories }\end{array}$ & Browse articles on similar topics from Cold Spring Harbor Protocols. \\
& Alignment of Sequences (46 articles) \\
& Alignment of Sequences, general (24 articles) \\
& Bioinformatics/Genomics, general (192 articles) \\
DNA:Protein Interactions (74 articles) & Genome Analysis (191 articles) \\
& Molecular Biology, general (1293 articles) \\
& Sequence Database Searching (38 articles) \\
& Yeast (288 articles) \\
\hline
\end{tabular}

